\title{
Über die Amputierung der Vertexfunktionen instabiler Teilchen
}

\author{
J. J. HenNing* \\ Nasionale Navorsingsinstituut vir Wiskundige Wetenskappe, Pretoria
}

Eingegangen am 15. November 1965

\begin{abstract}
The normalized form of the amputated vertex functions of an unstable particle is derived from the requirement that the general Ansatz (1) for the scattering amplitude of two stable particles with a resonance in the energy-variable is unitary in the energy interval below the threshold for three particle production. A Klein-Gordon equation for the unstable particle associated with the resonance is derived. The results a re applied to the creation of $\pi^{+}$and $\pi^{0}$ mesons in inelastic scattering processes.
\end{abstract}

\section{Einleitung}

Um den mathematischen Aufwand auf das Wesentliche zu beschränken, betrachten wir die Wechselwirkung spin- und ladungsloser Teilchen ( $A$-Teilchen). Bei der Streuung zweier $A$-Teilchen mit Masse $m_{a}$ kann ein $B$-Teilchen als instabiles Zwischenteilchen entstehen, sich fortpflanzen und wieder zerfallen. Wir sind der Ansicht, daß diese kausale Folge von Vorgängen durch eine Produktzerlegung

$$
\begin{gathered}
i T(\xi, \eta)={ }^{\operatorname{ein}}\left\langle p_{1} p_{2}|B(o)| O\right\rangle \Delta_{F}^{\prime}(\xi, B)\left\langle O|B(o)| p_{3} p_{4}\right\rangle^{\text {aus }} \Gamma(\xi) \\
\xi=\left(p_{1}+p_{2}\right)^{2}, \quad \eta=\left(p_{3}-p_{1}\right)^{2}
\end{gathered}
$$

des $T$-Matrixelements in

$$
{ }^{\text {ein }}\left\langle p_{1} p_{2} \mid p_{3} p_{4}\right\rangle^{\text {aus }}={ }^{\text {ein }}\left\langle p_{1} p_{2} \mid p_{3} p_{4}\right\rangle^{\text {ein }}+i \delta^{4}\left(p_{1}+p_{2}-p_{3}-p_{4}\right) T(\xi, \eta)
$$

beschrieben werden kann, wobei die Ausbreitungsfunktion des $B$-Teilchens durch

$$
\Delta_{F}^{\prime}(\xi, B)=(2 \pi)^{-4} \int_{-\infty}^{\infty} d^{4} x\langle O|T B(x) B(o)| O\rangle e^{\mathrm{i} \mathrm{px}}, \quad \xi=p^{2}
$$

definiert ist. Die reelle Funktion $\Gamma(\xi)$ wird eindeutig durch die Unitaritätsbeziehung für winkelunabhängige Streuung (oder ,,s-Streuung“)

$$
\operatorname{Im} T=\frac{\pi}{8} \sqrt{1+\frac{4 m^{2}}{\xi}}|T|^{2} \quad-9 m^{2}<\xi \leqq-4 m^{2}
$$

festgelegt.

Unsere Annahme über die Zerlegung (1) muß im folgenden Sinne verkunde.

* Adresse ab 1. Oktober 1965: Technische Hogeschool Delft, Afdeling Wis- 
standen werden: Beim Übergang zu lokalisierten Wellenpaketen für die stabilen $A$-Teilchen ergibt die Amplitude (1) für eine Ausbreitungsfunktion mit dem bekannten Pol im zweiten Blatt der Energievariablen genau das makroskopische Verhalten, das man als Erzeugung, Ausbreitung und Zerfall des instabilen $B$-Teilchens bezeichnen könnte [1], [2]. (Siehe auch die Erläuterungen über die Lokalisierung der stabilen Teilchen im nächsten Abschnitt.)

Auf Grund des TCP Theorems folgt im Intervall $-9 m^{2}<\xi \leqq$ $\leqq-4 m^{2}$ die Eigenschaft

$$
\begin{aligned}
V(\xi) & ={ }^{\operatorname{ein}}\left\langle p_{1} p_{2}|B(o)| O\right\rangle \\
& ={ }^{\text {aus }}\left\langle p_{1} p_{2}|B(o)| O\right\rangle^{*} \\
& =\left\langle O|B(o)| p_{1} p_{2}\right\rangle^{\text {aus }} \\
& =\left\langle O|B(o)| p_{1} p_{2}\right\rangle^{\text {ein }}\left[1+\frac{\pi}{4} \frac{1}{\sqrt{\xi\left(\xi+4 m^{2}\right)}} \int_{0}^{-\xi-4 m^{2}} i T(\xi, \eta) d \eta\right]
\end{aligned}
$$

für den hermiteschen Operator $B(x)$.

Die Substitution von (4) in (1) ergibt eine algebraische Gleichung für $T$. Die Lösung läßt sich sofort hinschreiben:

$$
i T=\frac{|V|^{2} \Delta_{F}^{\prime} \Gamma}{1-|V|^{2} \Delta_{F}^{\prime} \Gamma \frac{\pi}{4} \sqrt{1+\frac{4 m^{2}}{\xi}}}
$$

Die Substitution von (5) in (2) ergibt, mit

$$
\begin{gathered}
\Delta_{F}^{\prime}(\xi, B)=\frac{1}{2 \pi i} \int_{4 m^{2}}^{\infty} d v \frac{\varrho(v)}{v+\xi-i \varepsilon}, \\
\varrho(v)=\pi \sqrt{1+\frac{4 m^{2}}{v}}|V|^{2} \text { für } 9 m^{2}>v \geqq 4 m^{2}
\end{gathered}
$$

das einfache Ergebnis

$$
\Gamma=4 /\left|\Delta_{F}^{\prime}\right|^{2}
$$

\section{Die Wellenfunktion eines instabilen Teilchens}

Mit der unitären Näherung (7) kann die Streuamplitude (1) auch als Integral über das topologische Produkt zweier Minkowskischen Räume geschrieben werden:

$$
\begin{aligned}
{ }^{\text {ein }}\left\langle P_{1} P_{2} \mid P_{3} P_{4}\right\rangle^{\text {aus }}= & { }^{\text {ein }}\left\langle P_{1} P_{2} \mid P_{3} P_{4}\right\rangle^{\text {ein }}+ \\
& +\frac{(+4)}{(2 \pi)^{6}} \int_{-\infty}^{\infty} d^{4} x d^{4} y^{\text {ein }}\left\langle P_{1} P_{2}|B(x)| O\right\rangle_{\text {amp }} \times \\
& \times\langle O|T B(x) B(y)| O\rangle\left\langle O|B(y)| P_{3} P_{4}\right\rangle_{\text {amp }}^{\text {aus }},
\end{aligned}
$$


wobei die amputierten Vertexfunktionen durch

$$
{ }^{\text {ein }}\left\langle P_{1} P_{2}|B(x)| O\right\rangle_{\mathrm{amp}}={ }^{\operatorname{ein}}\left\langle P_{1} P_{2}|B(x)| O\right\rangle\left\{\left[2 \pi i \Delta_{F}^{\prime}(\xi, B)\right]^{*}\right\}^{-1}
$$

und

$$
\left\langle O|B(x)| P_{3} P_{4}\right\rangle_{\mathrm{amp}}^{\text {aus }}=\left\langle O|B(x)| P_{3} P_{4}\right)^{\text {aus }}\left[2 \pi i \Delta_{F}^{\prime}(\xi, B)\right]^{-1}
$$

definiert werden. Im kanonischen Modell mit der unrenormierten Lagrange-Dichte

$$
\begin{aligned}
\mathscr{L}= & -\frac{1}{2}\left(\partial^{\mu} A_{u}(x) \partial_{\mu} A_{u}(x)+m_{a, u}^{2} A_{u}^{2}(x)\right)(2 \pi)^{-3} \\
& -\frac{1}{2}\left(\partial^{\mu} B_{u}(x) \partial_{\mu} B_{u}(x)+m_{b, u} B(x)\right)(2 \pi)^{-3} \\
& -g_{u} A_{u}^{2}(x) B_{u}(x)(2 \pi)^{-3}
\end{aligned}
$$

wobei $\partial_{\mu}=\frac{\partial}{\partial x^{\mu}}=g_{\mu \nu} \partial^{\nu}, g^{\mu \nu}(-1)^{\delta_{o} \mu} \delta^{\mu \nu}$, kann die Ausbreitungsfunktion als

mit

$$
\Delta_{F}^{\prime}\left(p^{2}, B\right)=(2 \pi i)^{-1}\left[p^{2}+m_{b, r}^{2}+g_{r}^{2} \tilde{\Pi}\left(p^{2}\right)\right]^{-1}
$$

$$
\Pi\left(p^{2}\right)=\left(p^{2}+\lambda\right)^{2} \frac{\pi}{2} \int_{-\infty}^{-4 m_{a, r}^{2}} d v \frac{\varrho(v)}{\left(\nu-p^{2}+i \varepsilon\right)(\nu+\lambda)^{2}}
$$

geschrieben werden, wobei $\lambda$ eine für die Renormierung erforderliche Konstante ist [3]. Weiter hat $\varrho$ die Gestalt

$$
\varrho(v)=\sqrt{1+\frac{4 m_{a, r}^{2}}{v}}\left[1+\text { Glieder der Ordnung } g_{r}^{2}\right] \text {. }
$$

Die renormierten Massen im Quadrat werden durch

und

$$
\begin{aligned}
& m_{a, r}^{2}=m_{a, u}^{2}+\delta m_{a}, m_{b, r}^{2} Z_{2}=m_{b, u}^{2}+\delta m_{b}, \\
& \delta m_{a}=Z_{1} g_{r}^{2} \Sigma\left(-m_{a, r}^{2}\right), \delta m_{b}=Z_{2} g_{r}^{2} \Pi(-\lambda)+\lambda Z_{2} g_{r}^{2} \Pi^{\prime}(-\lambda), \\
& Z_{1}^{-1}=1-g_{r}^{2} \Sigma^{\prime}\left(-m_{a, r}^{2}\right)
\end{aligned}
$$

mit

$$
Z_{2}^{-1}=1-g_{r}^{2} \Pi^{\prime}(-\lambda)
$$

$$
\begin{aligned}
\Pi\left(p^{2}\right) & =\frac{-(2 \pi i)}{(2 \pi)^{4}} \int e^{i p x}\left\langle O\left|T A_{r}^{2}(x) A_{r}^{2}(o)\right| O\right\rangle d^{4} x, \\
\Sigma\left(p^{2}\right) & =\frac{-(2 \pi i)}{(2 \pi)^{4}} \int e^{i p x}\left\langle O\left|T A_{r}(x) B_{r}(x) A_{r}(o) B_{r}(o)\right| O\right\rangle d^{4} x,
\end{aligned}
$$

wobei $A_{r}(x)=\sqrt{Z_{1}} A_{u}(x), B_{r}(x)=\sqrt{Z_{2}} B_{u}(x)$ definiert. Die nackten Massen $m_{a, u}, m_{b, u}$ werden durch Regularisierung so definiert, daß $\Delta m>S$, $\Delta m=m_{b, r}^{2}-4 m_{a, r}^{2}$,

$$
S=-g_{r}^{2}\left(\lambda-4 m_{a, r}^{2}\right)^{2} \frac{\pi}{2} \int_{-\infty}^{-4 m_{a, r}^{2}} d \mu \frac{\varrho(\mu)}{\left(\mu+4 m_{a, r}^{2}\right)(\mu+\lambda)^{2}}
$$


Hieraus folgt dann:

1. Die gleichmäßige Konvergenz der Dämpfungsreihe, die zur störungstheoretischen Konstruktion des Propagators des instabilen Teilchens führt [4].

2. Die Instabilität des $B$-Teilchens oder die Existenz einer Nullstelle für $\left[\Delta_{F}^{\prime}\left(p^{2} ; B\right)\right]^{-1}$ im zweiten Blatt an der Stelle $p^{2}=-M^{2}+i \gamma$. Hierbei ist $M$ die experimentelle Masse des $B$-Teilchens (die Differenz zwischen $m_{b, r}^{2}$ und $M^{2}$ ist die ,Selbstmasse“, die infolge der Instabilität zustande kommt) während $\gamma^{-1}$ sich proportional zur Lebensdauer des Teilchens verhält.

Die $\left[2 \pi i \Delta_{F}^{\prime}\right]^{-1}$ Anwendung auf die Vertexfunktionen instabiler Teilchen entspricht hier der bekannten $K_{x}=\partial_{\mu} \partial^{\mu}-m_{b, r}^{2}$ Anwendung auf die Vertexfunktionen stabiler $B$-Teilchen im Konfigurationsraum.

Das Feynman-Diagram einer in dieser Weise amputierten Vertexfunktion hat kein Bein $\Delta_{F}^{\prime}\left(p^{2}\right)$ mehr, d. h. die Vertexfunktion $\left\langle O|B(o)| p_{3} p_{4}\right\rangle^{\text {aus }}=V(\xi)$ verliert ihren $\mathrm{Pol}\left[\xi+M^{2}-i \gamma\right]^{-1}$ im zweiten Blatt.

Wenn die $A$-Teilchen im Streuprozeß keine scharfen Impulse besitzen, sondern räumlich lokalisiert sind, lautet die Verallgemeinerung des S-Matrixelements (2):

$$
\begin{aligned}
& S\left(k_{1} k_{2} ; k_{3} k_{4}\right) \\
& \quad=\int_{-\infty}^{\infty} d^{3} P_{1} d^{3} P_{2} d^{3} P_{3} d^{3} P_{4} \Phi_{k_{1}}^{*}\left(P_{1}\right) \Phi_{k_{2}}^{*}\left(P_{2}\right) \Phi_{k_{3}}\left(P_{3}\right) \Phi_{k_{4}}\left(P_{4}\right) \times \\
& \quad \times{ }^{\operatorname{ein}}\left\langle P_{1} P_{2} \mid P_{3} P_{4}\right\rangle^{\text {aus }} .
\end{aligned}
$$

Der Ausdruck (9) entsteht aus (2) durch ,Lokalisierung“", d. h. durch die Substitutionen $e^{i p_{i} x} \rightarrow \int d^{3} p \Phi_{k_{i}}(p) e^{i p x}=\Psi_{k_{i}}(x) ; p_{0}=\sqrt{\mathbf{p}^{2}+m_{a}^{2}}$ wobei $k_{i}$ der mittlere Impuls des lokalisierten $i$-ten Teilchens ist [5]. Der Übergang von (2) zu (9) ist leicht verständlich, wenn man beachtet, daß eine Streuamplitude wie (2) oder (9) ipso facto als lineares Funktional über die Wellenfunktionen $e^{i p x}$ bzw. $\Psi_{k_{i}}(x)$ definiert wird [6]. Auf diese Weise entstehen die lokalisierten Vertexfunktionen (oder Formfaktoren)

und

$$
V_{1}(x)=\int_{-\infty}^{\infty} d^{3} P_{1} d^{3} P_{2} \Phi_{k_{1}}^{*}\left(P_{1}\right) \Phi_{k_{2}}^{*}\left(P_{2}\right)^{\text {ein }}\left\langle P_{1} P_{2}|B(x)| O\right\rangle_{\mathrm{amp}}
$$

$$
V_{2}(x)=\int_{-\infty}^{\infty} d^{3} P_{3} d^{3} P_{4} \Phi_{k_{3}}\left(P_{3}\right) \Phi_{k_{4}}\left(P_{4}\right)\left\langle O|B(x)| P_{3} P_{4}\right\rangle_{\mathrm{amp}}^{\mathrm{aus}}
$$

in dem Ausdruck

$$
\begin{aligned}
S\left(k_{1}, k_{2} ; k_{3}, k_{4}\right) & =\int_{-\infty}^{\infty} d^{3} P_{1} \ldots d^{3} P_{4} \Phi_{k_{1}}^{*}\left(P_{1}\right) \ldots \Phi_{k_{4}}\left(P_{4}\right)^{\text {ein }}\left\langle P_{1} P_{2} \mid P_{3} P_{4}\right\rangle^{\text {ein }}- \\
& -\frac{4}{(2 \pi)^{6}} \int_{-\infty}^{\infty} d^{4} x d^{4} y V_{1}(x) \Delta_{F}^{\prime}(x-y, B) V_{2}(y) .
\end{aligned}
$$


Die Wellenfunktionen im Impulsraum $\Phi_{k_{1}}(p), \Phi_{k_{2}}(p), \Phi_{k_{3}}(p), \Phi_{k_{4}}(p)$ sind so ausgeschmiert zu denken, daß $\Psi_{k_{i}}(x)$ nur in einer endlichen Umgebung der Minkowski-Weltlinien

$$
\mathbf{x}=\mathbf{V}^{i} x_{0} ; \quad i=1,2
$$

wesentlich von null verschieden sind [1], [2] während $\Psi_{k_{i}}(x), i=3,4$, nur in einer endlichen Umgebung der Linien

$$
\mathbf{x}=\mathbf{V}^{i}\left(x_{0}-\tau\right)
$$

$$
i=3,4
$$

ungleich null sind ${ }^{1}$. Bei Vorwärtsstreuung könnte man

$$
\Phi_{k_{3}}(p)=\Phi_{k_{1}}(p) e^{+i p_{0} \tau}, \Phi_{k_{4}}(p)=\Phi_{k_{2}}(p) e^{+i p_{0} \tau}, p_{0}=\sqrt{\mathbf{p}^{2}+m_{a}^{2}}
$$

ansetzen. Diesen Meßprozeß sieht man in Abb. 1. Unter den erwähnten Voraussetzungen für die Wellenpakete sind die Trägergebiete der Funktionen $V_{1}$ und $V_{2}$ mit den Überlappungsgebieten $A$ und $B$ in Abb. 1 identisch. Dies läßt sich etwa in der Störungstheorie, wo ein $\left\langle P_{1} P_{2}|B(o)| O\right\rangle_{\text {amp }}$ für ein kanonisches Modell als Potenzreihe in der Kopplungskonstante geschrieben werden kann, nachweisen.
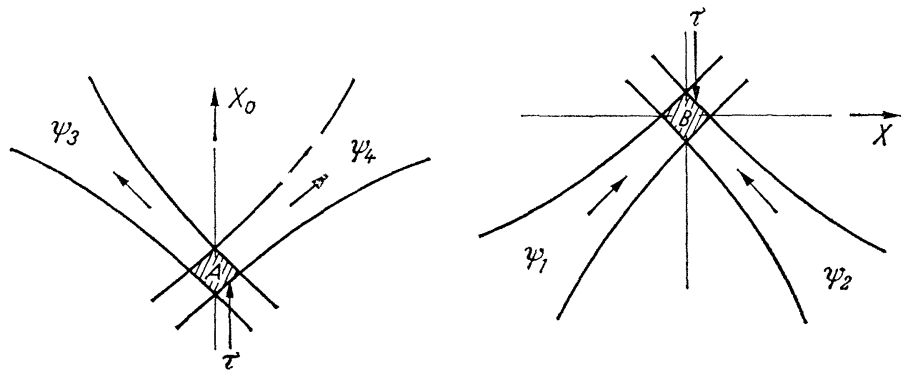

Abb. 1. Erzeugung und Zerfall eines instabilen Teilchens als ein im Konfigurationsraum beobachtbarer Streuvorgang

Die Lokalisierung der Erzeugungs- und Zerfallspunkte implizieren also, da $\beta$ in (10) die volle Ausbreitungsfunktion

$$
\Delta_{F}^{\prime}(x-y)=\Delta_{+}^{\prime}(x-y) \theta\left(x_{0}-y_{0}\right)+\Delta_{-}^{\prime}(x-y) \theta\left(y_{0}-x_{0}\right)
$$

durch $\Delta_{-}^{\prime}(x-y)$ ersetzt werden kann. Für raumzeitlich kausal getrennte Erzeugungs- und Zerfallspunkte kann (8) daher formal als

$$
\begin{aligned}
& { }^{\operatorname{ein}}\left\langle P_{1} P_{2} \mid P_{3} P_{4}\right\rangle^{\text {aus }}={ }^{\text {ein }}\left\langle P_{1} P_{2} \mid P_{3} P_{4}\right\rangle^{\text {ein }}+\frac{4}{(2 \pi)^{6}} \int_{-\infty}^{\infty} d^{4} x \int_{-\infty}^{\infty} d^{4} y \times \\
& \times{ }^{\operatorname{ein}}\left\langle P_{1} P_{2}|B(x)| O\right\rangle \cdot \overleftarrow{K}_{x} \Delta_{-}^{\prime}(x-y) \vec{K}_{y}\left\langle O|B(y)| P_{3} P_{4}\right\rangle^{\text {aus }}
\end{aligned}
$$

geschrieben werden, wobei

$$
\begin{aligned}
& { }^{\operatorname{ein}}\left\langle P_{1} P_{2}|B(x)| O\right\rangle \overleftarrow{K}_{y} \equiv \operatorname{ein}\left\langle P_{1} P_{2}|B(x)| O\right\rangle_{\text {amp }} \\
& ={ }^{\operatorname{ein}}\left\langle P_{1} P_{2}|B(x)| O\right\rangle\left[\left(2 \pi i \Delta_{F}^{\prime}(\xi)^{*}\right)\right]^{-1}, \xi=\left(P_{1}+P_{2}\right)^{2} \\
& =\left[\xi+m_{b, r}^{2}+g_{r}^{2} \Pi I^{*}(\xi)\right]^{\text {ein }}\left\langle P_{1} P_{2}|B(x)| O\right\rangle \text {. }
\end{aligned}
$$

1 Wir können hier nicht näher auf das Problem der statistischen Deutung der Klein-Gordon-Lösungen eingehen. Siehe hierzu u. a. die Artikel von Brenig u. HAAG [5] sowie WANDERS [1]. 
Wenn der Ausdruck (11) mit dem allgemeinen Ergebnis ( $T$ ist der „,chronologische“" Operator)

$$
\begin{aligned}
& \left\langle O\left|\bar{T} A\left(x_{1}\right) \ldots A\left(x_{i}\right) T A\left(x_{i+1}\right) \ldots A\left(x_{n}\right)\right| O\right\rangle \\
& =\left\langle O\left|\bar{T} A\left(x_{1}\right) \ldots A\left(x_{i}\right)\right| O\right\rangle\left\langle O\left|T A\left(x_{i+1}\right) \ldots A\left(x_{n}\right)\right| O\right\rangle+ \\
& +\int_{-\infty}^{\infty} d x \int_{-\infty}^{\infty} d y\left\langle O\left|\bar{T} A\left(x_{1}\right) \ldots A\left(x_{i}\right) B(x)\right| O\right\rangle \times \\
& \times\left(\overleftarrow{\square}^{2}-m_{b}^{2}\right) \Delta_{-}(x-y)\left(\vec{\square}_{y}^{2}-m_{b}^{2}\right)\left\langle O\left|T B(y) \ldots A\left(x_{n}\right)\right| O\right\rangle+ \\
& + \text { Beiträge mit Mehrteilchenzwischenzuständen }
\end{aligned}
$$

verglichen wird (hier ist das $B$-Teilchen stabil), sieht es so aus, als ob man in (8) (als Ersatz für stabile Zweiteilchenzustände) im S-Matrixelement ${ }^{\text {ein }}\left\langle p_{1} p_{2} \mid p_{3} p_{4}\right\rangle^{\text {aus }}$ über asymptotisch nicht freie auslaufende $B$-Teilchen-Zwischenzustände summiert hätte. Das instabile Teilchen wird daher formal durch die verallgemeinerte Klein-Gordon Gleichung

$$
\left[-\partial^{\mu} \partial_{\mu}+m_{b, r}^{2}+g_{r}^{2} \tilde{\Pi}\left(-\partial^{\mu} \partial_{\mu}\right)\right] \Phi(x)=0
$$

oder durch die Wellenfunktion

$$
\Phi(x)=\int_{C} d^{4} p \delta\left(p^{2}+m_{b, r}^{2}+g_{r}^{2} \tilde{\Pi}\left(p^{2}\right)\right) \widetilde{\Phi}(p) e^{i p x}
$$

beschrieben, wobei der Integrationsweg der Veränderlichen $p^{2}=p^{i} p_{i}$ durch die Nullstelle $z=-M^{2}+i \gamma$ im zweiten Blatt der Funktion $F(z)=z+m_{b, r}^{2}+g_{r}^{2} \tilde{\Pi}(z)$ geht. Komplexe Distributionen sind in diesem näheren Zusammenhang von NAKANISHI [7] untersucht worden, sie sind aber auch den Mathematikern schon längst bekannt [8].

\section{Einige realistische Fälle}

a) Betrachten wir nun den Vorgang in Abb. 2. Die Baryonen und Leptonenzahlerhaltungssätze sind erfüllt. Dann hat (8) die Form

$$
\begin{aligned}
{ }_{\text {ein }}^{\left\langle p p^{\prime} \mid D e^{+} v\right\rangle^{\text {aus }}} & =\frac{+4}{(2 \pi)^{6}} \int d^{4} x d^{4} y^{\text {ein }}\left\langle p p^{\prime}\left|\Phi^{+}(x)\right| D\right\rangle_{\text {amp }} \times \\
& \times\left\langle O\left|T \Phi^{+}(x) \Phi^{+}(y)\right| O\right\rangle\left\langle O\left|\Phi^{+}(y)\right| e, v\right\rangle_{\text {amp }}^{\text {aus }}
\end{aligned}
$$

wobei

und

$$
{ }^{\text {ein }}\left\langle p p^{\prime}\left|\Phi^{+}(x)\right| D\right\rangle_{\mathrm{amp}}={ }^{\operatorname{ein}}\left\langle p p^{\prime}\left|\Phi^{+}(x)\right| D\right\rangle\left[\left(2 \pi i \Delta_{F}^{\prime}(\xi)\right)^{*}\right]^{-1}
$$

$\left\langle O \mid \Phi^{+}(y)^{\prime} e, v\right\rangle_{\text {amp }}^{\text {aus }}=\left\langle O\left|\Phi^{+}(y)\right| e, v\right\rangle^{\text {aus }}\left[2 \pi i \Delta_{F}^{\prime}(\xi)\right]^{-1}, \xi=\left(p+p^{\prime}-p_{D}\right)^{2}$.

b) Photonenerzeugung eines $\pi^{0}$-Mesons. Das S-Matrix-Element für den Vorgang in Abb. 3 mit asymptotisch stabilen Teilchen hat in diesem Fall die Gestalt

$$
\begin{aligned}
\operatorname{ein}\left\langle\gamma K_{1} \mid \gamma_{1} \gamma_{1} K_{2}\right\rangle^{\text {aus }} & =\frac{+4}{(2 \pi)^{6}} \int_{-\infty}^{\infty} d^{4} x \int_{-\infty}^{\infty} d^{4} y^{\text {ein }}\left\langle\gamma K_{1}|\Phi(x)| K_{2}\right\rangle_{\text {amp }} \times \\
& \times\langle O|T \Phi(x) \Phi(y)| O\rangle\left\langle O|\Phi(y)| \gamma_{1} \gamma_{2}\right\rangle_{\text {amp }}^{\text {aus }}
\end{aligned}
$$


mit

$$
\begin{aligned}
\operatorname{ein}\left\langle\gamma K_{1}|\Phi(x)|\right. & \left.K_{2}\right\rangle_{\mathrm{amp}} \\
& ={ }^{\operatorname{ein}}\left\langle\gamma K_{1}|\Phi(x)| K_{2}\right\rangle\left[\left[2 \pi i \Delta_{F}^{\prime}\left(\left(p_{\gamma}+p_{K_{1}}-p_{K_{2}}\right)^{2} ; \pi^{o}\right)\right]^{*}\right]^{-1}
\end{aligned}
$$

und

$\left.\left\langle O|\Phi(y)| \gamma_{1} \gamma_{2}\right\rangle_{\text {amp }}^{\text {aus }}=\left\langle O|\Phi(y)| \gamma_{1} \gamma_{2}\right\rangle^{\text {aus }}\left[2 \pi i \Delta_{F}^{\prime}\left(\left(\gamma_{1}+\gamma_{2}\right)^{2} ; \pi^{o}\right)\right)\right]^{-1}$

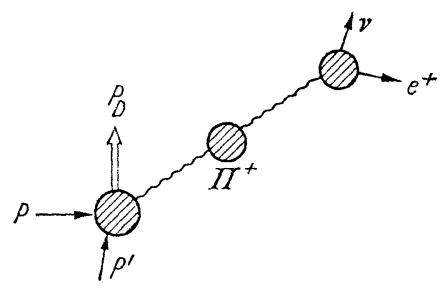

Abb. 2. Erzeugung und Zerfall eines positiven Mesons

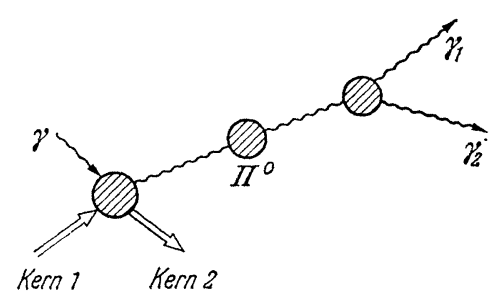

Abb. 3. Photo-Erzeugung und Zerfall eines neutralen Mesons

Die Vertexfunktionen $\left\langle O\left|\Phi^{+}(y)\right| e, v\right\rangle^{\text {aus }}$ und $\left\langle O|\Phi(y)| \gamma_{1} \gamma_{2}\right\rangle^{\text {aus }}$ sind mit dispersionstheoretischen Methoden von Goldberger und Treiman [9] untersucht worden, während u. a. Glaser u. Ferrell [10] die letztgenannte Funktion im Rahmen eines einfachen kanonischen Modells explizit berechnet haben.

\section{Schlußbemerkungen}

Unsere Ausführungen in den Abschnitten II und III zeigen, welche Rolle instabile Teilchen in einer $S$-Matrix Theorie spielen. Bei räumlich stark getrennten Erzeugungs- und Zerfallsprozessen von instabilen Teilchen kann ihr voller Propagator im Sinne der Methode mit Feynmandiagrammen benutzt werden. Bei der oben diskutierten Amputierung der Scheitelfunktionen bleiben die Näherungen unitär. Wir sind hierbei lediglich von den folgenden drei Eigenschaften ausgegangen:

(1) Der kausale Zusammenhang zwischen Erzeugung und Zerfall des instabilen Teilchens, oder ,Makrokausalität".

(2) Die Unitarität des S-Operators.

(3) Die Invarianz gegenüber eigentlichen Lorentztransformationen. Bei der Ableitung der Wellenfunktion des instabilen Teilchens war es jedoch wichtig, daß man ein kanonisches Modell mit Wechselwirkungsglied zu Hilfe nimmt. Aus der Wellengleichung des $B$-Teilchens folgert man, daß die Wechselwirkung des instabilen Teilchens mit seinen Zerfallsteilchen in der renormierten Kopplungskonstante bilinear ist, und nicht linear, wie aus einer einfachen $d e$-Quantisierung des $B$-Feldes zu erwarten wäre. 
222 J.J. Henning: Über die Amputierung der Vertexfunktionen instabiler Teilchen

\section{Anhang}

Über die Bildung von Operatoren für instabile Teilchen aus den Operatoren der stabilen Zerfallsteilchen

Eine reine S-Matrix-Theorie wird vollständig durch die Vakuumserwartungswerte von Produkten von lokalen Operatoren gegeben. In diesen Funktionen treten nur die Operatoren der stabilen Elementarteilchen auf. Es ist jedoch zweckmäßig, in vielen Fällen (wie z. B. in den zwei Beispielen in Abschnitt III) Operatoren für instabile Teilchen in die Theorie einzuführen. Es wäre heuristisch sehr wertvoll, diese Operatoren durch Produktbildung aus den lokalen Operatoren der stabilen Zerfallsteilchen zu gewinnen. So könnte man für das Neutron (als instabiles Teilchen) den Operator

$$
\Psi_{n}(x)=\gamma^{\mu} \Psi_{p}(x)\left(\bar{\Psi}_{v}(x) \gamma_{\mu} \Psi_{e}(x)\right)
$$

bilden, wobei $\Psi_{p}(x), \Psi_{v}(x), \Psi_{e}(x)$ die Operatoren für Proton, Antineutrino und Elektron sind. In dem Ausdruck (12) benutzen wir die Bezeichnungsweise von JAUCH u. RoHRLICH [11] für die Bispinoren $\Psi(x), \bar{\Psi}(x)$. Die Lebensdauer des Neutrons kann dann [3], [12] aus der Stelle des Pols in der analytischen Fortsetzung der Spektralfunktionen in der Lehmannschen Darstellung

$$
\begin{array}{r}
\left\langle O\left|T \gamma_{\mu} \Psi_{p}(x)\left(\bar{\Psi}_{\nu}(x) \gamma^{\mu} \Psi_{e}(x)\right) \gamma^{\sigma} \bar{\Psi}_{p}(o)\left(\bar{\Psi}_{e}(o) \gamma_{\sigma} \Psi_{\nu}(o)\right)\right| O\right\rangle \\
=\int_{-\infty}^{\infty} d^{4} p \int_{\left(m_{e}+m_{p}\right)}^{\infty} d \alpha \frac{\varrho_{1}\left(\alpha^{2}\right) I+\left(\gamma_{\mu} p^{\mu}+\alpha\right) \varrho_{2}\left(\alpha^{2}\right)}{p^{2}+\alpha^{2}-i \varepsilon} e^{-i p x}
\end{array}
$$

bestimmt werden.

\section{Literatur}

[1] Wanders, G.: Nuovo Cimento 14, 168 (1959).

[2] Yамамото, K.: Progr. Theoret. Phys. (Kyoto) 10, 857 (1959).

[3] Henning, J. J.: Z. Physik 177, 277 (1964).

[4] - Z. Physik 178, 253 (1964).

[5] Brenig, W., u. R. HaAG: Fortschr. Physik 4, 183 (1959).

[6] Lehmann, H., K. Symanzik u. W. ZimmermanN : Nuovo Cimento 1, 205 (1955).

[7] Nakanishi, N.: Progr. Theoret. Phys. (Kyoto) 19, 607 (1958).

[8] Gel'fand, I. M., u. G. E. SchILow (ShILOv): Generalized functions, Vol. I. Übersetzt von E. Saletan. New York: Academic Press 1964. Anhang B.

[9] Goldberger, M., u. S. Treiman: Nuovo Cimento 9, 451 (1958).

[10] Glaser, V., u. R. A. Ferrell: Phys. Rev. 121, 886 (1961).

[11] JAUCH, J. M., u. F. RoHRLICH: The theory of photons and electrons. Reading, Pa.: Addison Wesley Publishing Co. 1955.

[12] Peierls, R. E.: Proceedings of the 1954 Conference on Nuclear and Meson Physics, p. 296. New York: Pergamon Press. 